\title{
AN IMAGE INTENSIFIER-SCINTILLATOR DEVICE FOR DETERMINATION OF PROFILES AND IMAGES OF WEAK BEAMS OF IONIZING PARTICLES OR X-RAYS $\dagger$
}

\author{
LAWRENCE W. JONES and MARTIN L. PERL
}

Harrison M. Randall Laboratory of Physics, The University of Michigan, Ann Arbor, Michigan

Received 17 January 1960 An instrument for determining the shape and intensity distri-
bution of particle and X-ray beams has been developed using an
image intensifier tube together with a bundle of filaments of scintillator. A description of the use of such a device in high

\section{Introduction}

It is possible to construct a monitor for determining the intensity profiles of weak beams of highenergy charged particles or X-rays by combining filamentary scintillators with an image converter tube. Such a device, which we will refer to as a "Channeled Particle-Image Converter" or CPIC, has been constructed and used by the authors in meson beams from the Bevatron. A discussion of the problems of such monitoring and of existing methods in these fields follows. The CPIC as constructed is described along with calculations of conversion and detection efficiencies. Further extensions of this new instrument and other applications particularly to high energy X-radiology conclude the paper.

In high energy physics, beam transport systems consisting of quadrupoles, bending magnets, and electromagnetic separators are generally set in position according to calculations and measurements on the separate components $\left.{ }^{1}\right)$. The various operating currents are then adjusted about their predicted values to optimize the beam intensity and spatial distribution at the position of either a target of liquid hydrogen (or some other material) or the last slit of a beam separator. In order to monitor the beam profile in the target plane a small scintillation counter is frequently used, moving it back and forth and comparing its counting rate

f Supported by the U. S. Office of Naval Research. energy, low intensity, secondary meson and proton beams is given. It is shown that the sensitivity of this instrument represents a significant improvement over alternative techniques.

with a suitable monitor. Weigand ${ }^{2}$ ) has constructed a more elaborate profile monitor consisting of a row of scintillation counters connected to an oscilloscope in such a way that the oscilloscope trace gives beam intensity versus position in one dimension perpendicular to the beam axis. These methods are inconvenient and of limited resolution. The purpose of the monitor described here is to give a two-dimensional presentation of such a beam intensity distribution in weak particle beams.

A second area of usefulness of the same device is in the high-energy industrial X-radiography of thick castings or other objects, where it appears that considerable improvements over present technology are possible.

\section{Principle of Operation}

A large sheet of scintillator perpendicular to the beam axis viewed from a distance by eye or by a television or camera system has been used in several instances ${ }^{3,4}$ ) for viewing intense beams (e.g., $10^{5}$ minimum ionizing particles per $\mathrm{cm}^{2}$ per second or greater). However, the poor light collection efficiency and relatively poor efficiency of the detectors

1) H. K. Ticho, International Conference on High Energy Accelerators and Instrumentation - CERN 1959, Proceedings (Geneva, 1959) p. 387.

2) C. Weigand (private communication).

3) R. D. Sard, Proceedings of an International Conference on Instrumentation for High Energy Physics Berkeley, 1960 (Interscience publishers, New York, 1961).

4) J. S. Pruitt, Nucleonics 13 No. 8 (1955) 26. 
used restricts this method to beams generally more intense than the secondary particle beams of accelerators such as the Bevatron and Cosmotron ${ }^{5}$ ).

By placing the scintillator very close to the large diameter photocathode of an image intensifier tube,

\section{Experimental Device and Operation}

Using this principle, a CPIC was assembled as indicated in figs. 1, 2, and 3. The image intensifier used was a Westinghouse WL7257 employing an S1l cathode and PI1 phosphor; the cathode dia-

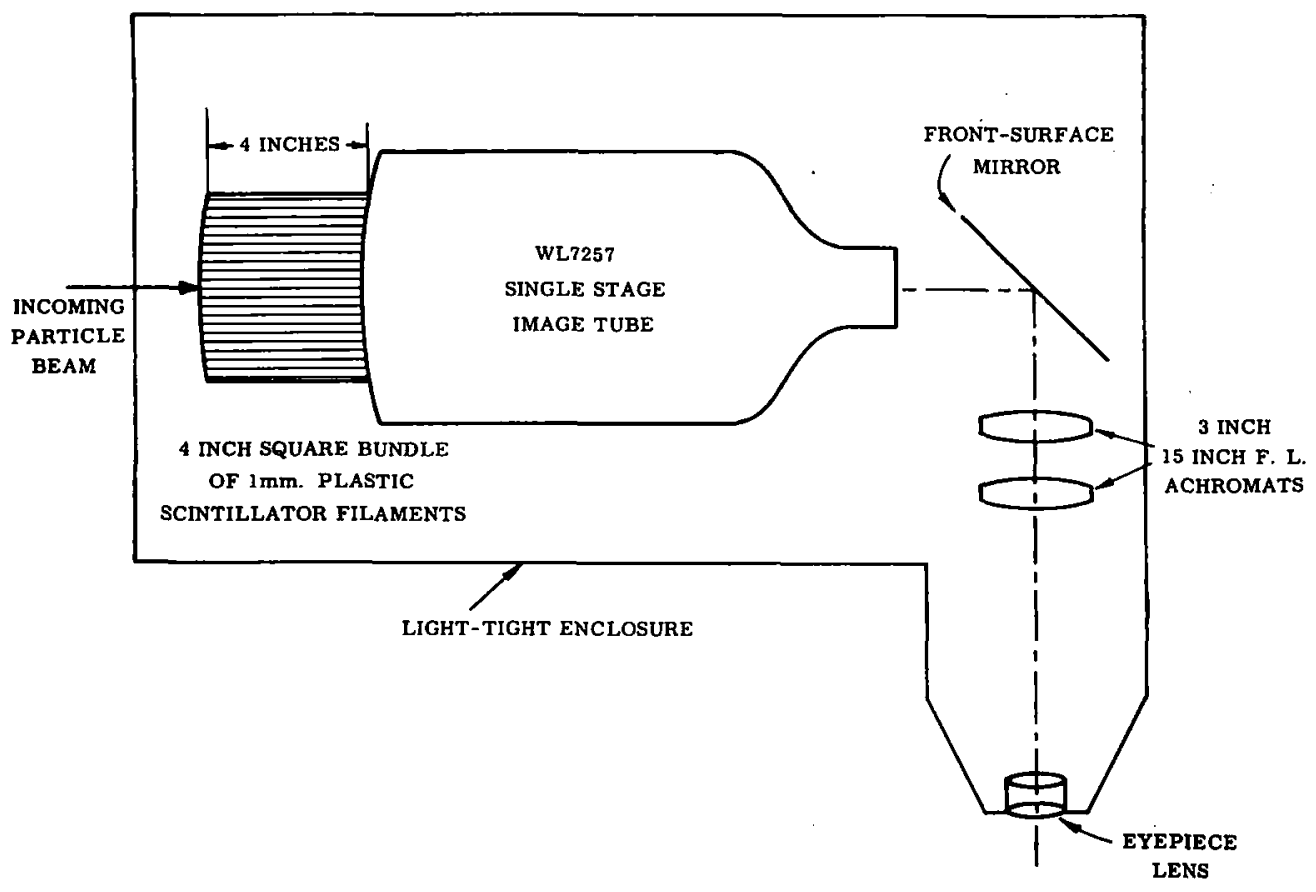

Fig. 1. Schematic diagram of Beam Profile Monitor as assembled for early tests. The periscope optics allows the observer to be out of the beam.

the light collection and detection efficiency are both greatly improved, however the spatial resolution is poor, comparable to the thickness of the scintillator used. Briefly, an image intensifier (or image converter) tube consists of a photocathode, accelerating and focusing electrodes, and a phosphor-screen anode. Thus an image focused on the cathode is reproduced with increased brightness at the phosphor, where the brightness gain may be $10^{2}$ to $10^{4}$, depending on the image tube characteristics. The resolution can then be improved by constructing the scintillator of a bundle of filaments ${ }^{6}$ ) with its axis parallel to the beam. The filaments channel the light by total internal reflection, thus permitting use of a converter (scintillator) up to several centimeters thick. The resolution in this case is determined by the filament diameter and the thickness of the cathode envelope of the image tube. meter is 5 inches, the phosphor diameter 1 inch, the cathode efficiency $8 \%$ (to blue quanta), and the quantum gain about 80 at 30 kilovolts operating voltage. The area demagnification from cathode to anode is $25: 1$ so that the above quantum gain corresponds to a brightness increase of 3000 . The scintillator was in the form of $1 \mathrm{~mm}$-diameter plastic filaments each $10 \mathrm{~cm}$ long arranged in a $10 \mathrm{~cm}$ square lucite frame. The tube was enclosed in a magnetic shield and a light-tight box. A viewing periscope allowed an observer to see the anode without undue radiation exposure in the beam proper.

A quantitative estimate of the performance of

5) In a modification of Sard's apparatus, Kerth has replaced the polaroid film by an image orthicon ( $Z$ 5294) viewing a sheet of sodium iodide through an $f / 1.1$ lens and reports a sensitivity possibly as high as $10^{4}$ particles per $\mathrm{cm}^{2}$ per second. 


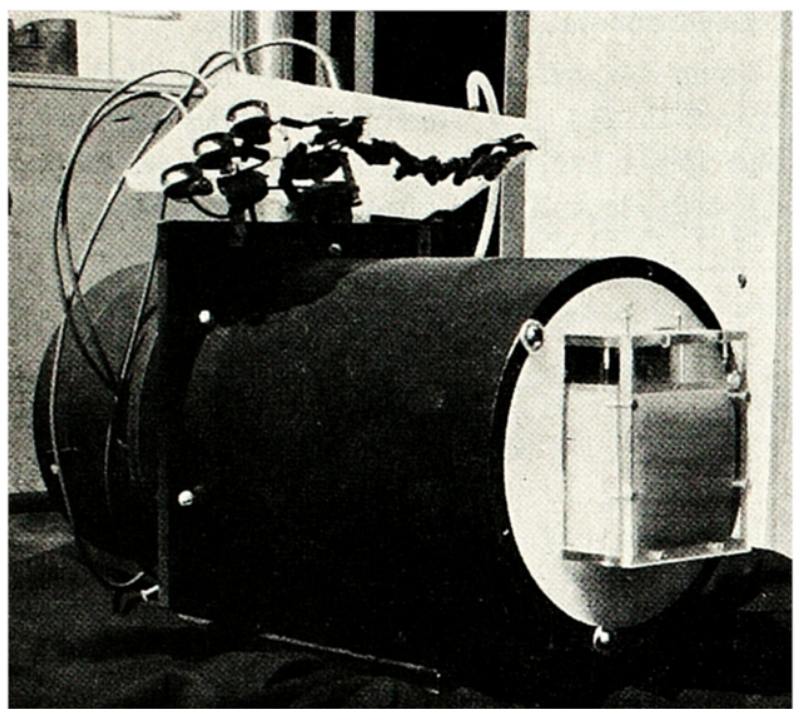

Fig. 2. Photograph of the image tube and scintillator assembly. The image tube is within the iron magnetic shield, the scintillator bundle is visible on the right. A resistor voltage divider network for supplying the focusing electrode voltages is mounted above the assembly.

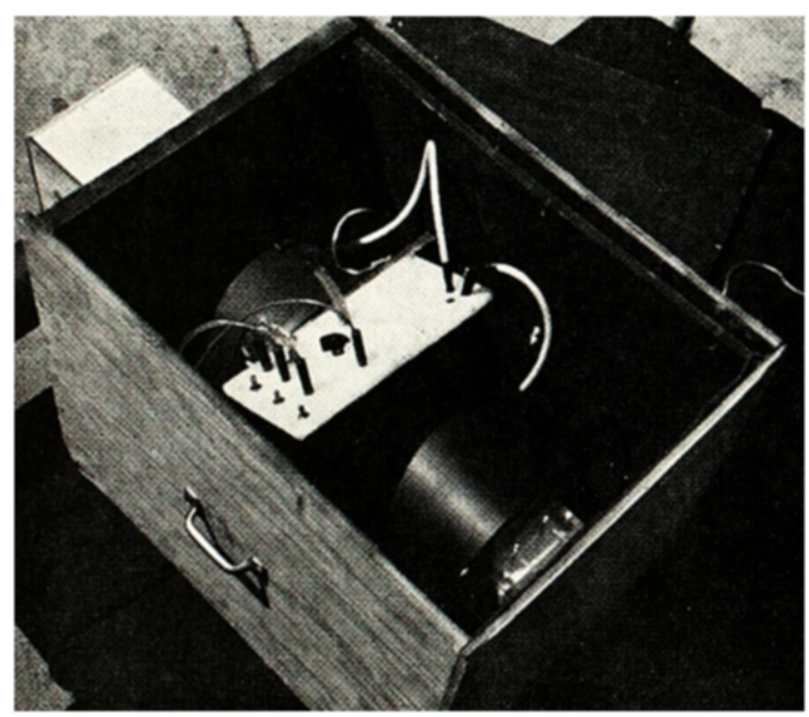

Fig. 3. Photograph of the image tube and scintillator assembly in place within its light-tight box (cover removed).

this system may be made as follows. A minimumionizing particle produces about $1.6 \times 10^{4}$ quanta per $\mathrm{cm}$ of path in plastic scintillator, and about $20 \%$ of these quanta are "piped" to each end by total internal reflection ${ }^{6}$ ) (assuming perfect surfaces on the filaments). Due to light losses from imperfect filaments, the light reaching one end of a $10 \mathrm{~cm}$ filament might be only $10^{4}$ quanta. These traverse the $\frac{1}{8}$ inch cathode envelope and produce photoelectrons from the image tube cathode. From the measured image tube gain, about $8 \times 10^{5}$ quanta will be produced at the anode phosphor from each particle traversing the scintillator.

The WL7257 has an area demagnification of 25 from cathode to phosphor, so that an incident flux of $N$ minimum-ionizing particles per $\mathrm{cm}^{2}$ per second will produce about $2 \times 10^{7} \mathrm{~N}$ photons per $\mathrm{cm}^{2}$ per second at the anode. The resolution of this system is limited by the cathode envelope thickness to about $3 \mathrm{~mm}$ (at the cathode).

This system was used with a beam of $1.5 \mathrm{GeV} / \mathrm{c}$ negative particles (mesons) from the Bevatron. At a flux of 1000 particles per $\mathrm{cm}^{2}$ per pulse the intensity distribution was readily visible, the beam center could be located, and the characteristic pattern of the beam due to the combined effects of bending magnet dispersion and chromatic aberration of the quadrupole was apparent. Measurements with scintillation counters were in agreement with the monitor observations.

\section{Further Developments}

The usefulness of this first device may be improved in several ways. First, the beam intensity pattern appeared to the observer on an otherwise dark background with no reference to space coordinates in the laboratory. It is planned to add fiducial lines to the image tube input on which the eye can focus between beam pulses and to which the beam intensity pattern may be referred.

The other factors which could be improved are the sensitivity of the system and a quantitative presentation of the beam intensity pattern to supplement the qualitative visual image. By

6) R. J. Potter and R. E. Hopkins, Proceedings of the Seventh Scintillation Counter Symposium, IRE Transactions NS-7, No. 2-3 (1960) p. 150;

G. T. Reynolds, p. 115, ibid; other relevant discussions of filament scintillators and image intensifier tubes are to be found in this volume; in session 7 in ref. ${ }^{1}$ ); in Advances in Electronics and Electron Physics 12 (Academic Press, New York, 1960); and in Proceedings of the Image Intensifier Symposium of the U.S. Army Engineers Research and Development Laboratory (1958) available through the Office of Technical Services, Washington, D.C. 
replacing the filaments of plastic scintillator with filaments of thalium-activated sodium iodide or cesium iodide an increase of approximately a factor of ten in sensitivity would be realized, since the light produced per unit length of track in these materials is an order of magnitude greater than in plastic. However the fabrication of such filaments is more difficult than with plastic, and they are thus not readily available.

Coupling the image tube output to an image orthicon would allow remote viewing of the image as well as increased sensitivity. For example, a pair of $f / 0.87$ Super Farron lenses would transmit about $15 \%$ of the light from the image tube anode to the cathode of an image orthicon, or about $10^{5}$ quanta per incident particle in the scintillator in the example above. The image tube resolution is 10-15 line pairs per $\mathrm{mm}$, so that the light from each filament is spread over an area determined by the thickness of the cathode envelope, the filament diameter, and the image tube demagnification. In the present case this is about $10^{-2} \mathrm{~cm}^{2}$ or less. For $N$ particles per $\mathrm{cm}^{2}$ incident on the scintillator, there would be $3 \times 10^{6} \mathrm{~N}$ photons per $\mathrm{cm}^{2}$ on the orthicon cathode.

A conventional image orthicon (RCA 5820) requires at least $2 \times 10^{9}$ photons per $\mathrm{cm}^{2}$ per second $\dagger$ to present to the eye a useful picture, thus such a system would have a threshold of $10^{3}$ beam particles per $\mathrm{cm}^{2}$ per seond. The photon density from a single beam particle would be about $10^{7}$ per $\mathrm{cm}^{2}$ at the orthicon cathode, thus at less than 1000 beam particles per $\mathrm{cm}^{2}$ second the problem becomes one of detecting single beam particles. An intensifier image orthicon (RCA C-74036) should have sufficient sensitivity to record single beam particles?). A thin-target orthicon (G.E. type $Z$ 5294) has a sensitivity intermediate between the conventional and intensifier orthicons, and a system employing such a G.E. orthicon together with the

\footnotetext{
$\dagger$ In addition to this rate threshold, there is also a total threshold because of the behavior of the eye and the properties of the television system. Not only must the rate be greater than $2 \times 10^{9}$ photons $/ \mathrm{cm}^{2} \mathrm{sec}$ but also the total photons $/ \mathrm{cm}^{2}$ must be greater than $4 \times 10^{8}$. In these discussions it is important to distinguish between total integrated light (as relevant in photographic recording), light per second, light per pulse (of accelerators such as the Bevatron), and light per frame of television scan.
}

components described in section 3 is currently being assembled at the Lawrence Radiation Laboratory ${ }^{8}$.

The quantitative profile of the beam may be readily observed by disecting a single video scan line from the orthicon signal and displaying this video amplitude on an oscilloscope $\mathrm{e}^{9}$ ).

As alternatives to the image tube-intensifier image orthicon system, one might have a two-stage image intensifier following the first tube with its output viewed by eye, an image orthicon, or even a vidicon. This system would also have ultimate sensitivity, in that it would permit detection of single beam particles.

Replacing the television tube by fast film (Kodak Royal-X Pan or Polaroid type 47) in the focal plane of the $f / 0.87$ lenses would permit a photographic record of particle fluxes as an alternative to visual observation. The threshold sensitivity of such film corresponds to about 1000 beam particles per $\mathrm{cm}^{2}$ incident.

The resolution of the system might be improved by using finer and shorter scintillator filaments and an image intensifier with either a very thin cathode envelope or a cathode window made of glass filaments. Both concepts have been incorporated into experimental tubes of the WL 7257 type. The advent of scintillating glass ${ }^{10}$ ) fabricated into filaments would permit finer filaments than possible with plastic.

It is also possible to use Cerenkov radiation in glass or plastic non-scintillating filaments, although in this case the system sensitivity is reduced an order of magnitude. In cases where a large neutron background exists, for example, this method of detection might be convenient.

Image tubes with cathodes 8 to 10 inches in diameter are being fabricated for medical radiology and will become available as light amplifiers for applications such as this.

7) B. A. Bang, Proceedings of the 5th International Congress on High Speed Photography of the Society for Motion Picture and Television Engineers, Washington, D.C. (1960) (to be published).

$\left.{ }^{8}\right)$ Dale Dickinson (private communication).

9) L. T. Kerth, (private communication).

10) R. J. Ginther, Proceedings of the Seventh Scintillation Counter Symposium, p. 28. 


\section{X-ray Monitoring}

This same combination of scintillation filaments and image intensifier should prove very useful in high-energy X-radiography. Currently, steel castings and other large objects are examined by directing the bremsstrahlung beam of a betatron directed through the object onto a sheet of $\mathrm{X}$-ray film which in turn is usually mounted behind a thin sheet of Jead as an intensifying screen. Exposures of 30 or 40 minutes are frequently necessary for sufficient detail on the film, primarily due to the poor efficiency of the film for high-energy electrons and $\mathrm{X}$-rays. Pruit ${ }^{3}$ ) has used a large sodium iodide crystal with a fast lens and film, however, the resolution and sensitivity of his method was not adequate for most purposes. For lower X-ray energies, a zinc sulfide-type phosphor screen is placed adjacent to the photocathode of an image intensifier tube, and it is indeed for this use that the WL 7257 type of image tube was developed11). However at energies well above the $\mathrm{K}$ absorption edge of zinc, the conversion efficiency of the thin phosphor is poor, and such devices are not generally useful above one $\mathrm{MeV}$.

The CPIC as described above, using sodium iodide filaments, or using plastic scintillator filaments behind a thin lead sheet, would be capable of converting $10-20 \%$ of all incident $X$-rays in the energy range above one $\mathrm{MeV}$. The resulting conversion electrons would produce light with the efficiencies computed above for minimum ionizing particles. Thus a single intensifier tube system would permit detection of X-ray fluxes of $10^{3}$ to $10^{4}$ quanta per $\mathrm{cm}^{2}$ per second, and a system employing also an intensifier image orthicon or a second image tube should be capable of detecting every converted X-ray photon above one $\mathrm{MeV}$.

Using glass scintillator filaments down to 10 microns diameter the technique might permit improvements in sensitivity and resolution also in the energy region below $100 \mathrm{keV}$. In fact such an $\mathrm{X}$-ray converter screen of scintillator filaments might be generally useful aside from the image intensifier for visual observation (fluoroscopy) or photographic recording of X-ray images at higher intensities.
The limiting resolution of such X-ray intensifier systems is determined by the transverse range of the conversion electrons (from compton scattering, photoeffect, or pair production) in the scintillator filaments. At energies above one $\mathrm{MeV}$ this is of the order of a millimeter, roughly independent of energy. At energies less than $500 \mathrm{keV}$ the resolution would improve. A corresponding limit to the resolution with high energy $\mathrm{X}$-rays arises from compton-scattered $\mathrm{X}$-rays from the object under study, and sets a limit to the resolution possible with any detection system.

A further use of the $\mathrm{X}$-ray detector as a "pinhole camera" for extended sources of X-ray or gamma radiation has been considered. Here an image tube with a filamentary $\mathrm{X}$-ray converter would be mounted in a lead or tungsten canister perhaps $1 \frac{1}{2} \mathrm{~cm}$ thick. A hole in the end of the canister opposite the converter would image the extended source on the filament array. All of the considerations discussed above apply. Such a device should find application in medicine, tracer techniques, and radiobiological safety.

\section{Summary}

A device for determining the intensity distribution of high energy particle beams consisting of scintillator filaments against the cathode envelope of an image intensifier tube has been built and successfully operated. Straight-forward extension of this technique will allow the sensitivity to be extended to the point where single particles of weak beams are resolved. The same technique carries over into X-ray detection, where corresponding advantages should be realized, particularly at X-ray energies above one $\mathrm{MeV}$. Thus images consisting of radiation quanta should be detectable for quanta from $100 \mathrm{keV}$ to arbitrarily high energies and at a level where at least every tenth quantum is seen.

\section{Acknowledgement}

It is a pleasure to acknowledge the hospitality of the Lawrence Radiation Laboratory during the testing of this monitor.

11) J. W. Coltman, Radiology 51 (1948) 359. 